\title{
Atlas Guided Identification of Brain Structures by Combining 3D Segmentation and SVM Classification
}

\author{
Ayelet Akselrod-Ballin ${ }^{1}$, Meirav Galun ${ }^{1}$, Moshe John Gomori², \\ Ronen Basri ${ }^{1}$, and Achi Brandt ${ }^{1}$ \\ ${ }^{1}$ Dept. of Computer Science and Applied Math, Weizmann Institute of Science, \\ Rehovot, Israel \\ ${ }^{2}$ Dept. of Radiology, Hadassah University Hospital, Jerusalem, Israel
}

\begin{abstract}
This study presents a novel automatic approach for the identification of anatomical brain structures in magnetic resonance images (MRI). The method combines a fast multiscale multi-channel three dimensional (3D) segmentation algorithm providing a rich feature vocabulary together with a support vector machine (SVM) based classifier. The segmentation produces a full hierarchy of segments, expressed by an irregular pyramid with only linear time complexity. The pyramid provides a rich, adaptive representation of the image, enabling detection of various anatomical structures at different scales. A key aspect of the approach is the thorough set of multiscale measures employed throughout the segmentation process which are also provided at its end for clinical analysis. These features include in particular the prior probability knowledge of anatomic structures due to the use of an MRI probabilistic atlas. An SVM classifier is trained based on this set of features to identify the brain structures. We validated the approach using a gold standard real brain MRI data set. Comparison of the results with existing algorithms displays the promise of our approach.
\end{abstract}

\section{Introduction}

Accurate classification of magnetic resonance images (MRI) is essential in many neuroimaging applications. Quantitative analysis of anatomical brain tissue such as white matter (WM), gray matter (GM) and cerebrospinal fluid (CSF) is important for clinical diagnosis, therapy of neurological diseases and for visualization and analysis $([1,2],[3])$. However, automatic segmentation of MRI is difficult due to artifacts such as partial volume effect (PVE), intensity nonuniformity (INU) and motion. The INU artifact also referred to as inhomogeneity or shading artifact causes spatial inter-scan variation in the pixel intensity distribution over the same tissue classes. It depends on several factors but is predominantly caused by the scanner magnetic field.

Numerous approaches have been developed for Brain MRI segmentation (see surveys [1, 4, [5, [6]). Low-level classification algorithms (which we compare to in sec. 3) typically involve common unsupervised algorithms including k-means 
and fuzzy c-means ([2],4]). Yet, low level techniques that exploit only local information for each voxel and do not incorporate global shape and boundary constraints are limited in dealing with the difficulties in fully automatic segmentation of brain MRI. Deformable models have also been proposed in [7] to find coupled surfaces representing the interior and exterior boundary of the cerebral cortex. These techniques benefit from consideration of global prior knowledge about expected shape yet their limitations come from dependence on initialization and from the computation time required for 3D segmentation.

Statistical approaches, which classify voxels according to probabilities based on the intensity distribution of the data, have been widely used [8]. These approaches typically model the intensity distribution of brain tissues by a Gaussian mixture model. Given the distribution, the optimal segmentation can be estimated by a maximum a posteriori (MAP), or a maximum likelihood (ML) formulation $([2,[3])$. The expectation maximization (EM) is a popular algorithm for solving the estimation problem. It has been applied to simultaneously perform brain segmentation and estimate the INU correction [9]. The EM framework has been extended to account for spatial considerations by including a Markov Random Field [10] and by utilizing a brain atlas [11.

This paper introduces a fully automatic method to identify brain structures in MRI, utilizing the 3D segmentation framework presented in [12, which extends the algorithm presented in $(13,, 14)$ to handle 3D multi-channel anisotropic MRI data. Our work combines the fast multiscale segmentation algorithm with a support vector machine (SVM) classifier based on a novel set of features. We incorporate prior knowledge of anatomic structures using an MRI brain atlas. In addition to these priors a set of regional features are computed for each aggregate, which includes intensity, texture, and shape features, accumulated during the aggregation process.Unlike existing studies our approach does not involve explicit correction of magnetic field inhomogeneities. We validate our approach by applying our method to a standard data base with varying bias field and compare our results to existing algorithms.

The paper is organized as follows. Section 2 describes the segmentation, feature extraction and classification process. Comparative experimental results for automatic detection of the major brain anatomical tissues are shown in section 3 . Concluding remarks are provided in section 4 .

\section{Method}

\subsection{Segmentation}

Our method begins with utilizing the segmentation algorithm presented in [12. This algorithm has extended the 2D segmentation algorithm developed for natural images ([13, 14]) to apply it to 3D multi-channel anisotropic MRI data. The segmentation scheme is described briefly below (for more details see [12, [13, ,14]).

The method, which is derived from algebraic multigrid (AMG) [15, starts by assembling together adjacent voxels into small aggregates based on intensity similarity, each voxel being allowed to belong to several aggregates with different 
association weights. These aggregates are then similarly assembled into larger aggregates, then still larger aggregates, etc. The affiliations between aggregates are based on tunable statistical measures, which are called features. Features obtained for small aggregates at a fine level affect the aggregation formation of larger aggregates at coarser levels, according to feature resemblance (see 2.2). In this multiscale Segmentation by Weighted Aggregation (SWA), a pyramid (hierarchy) of aggregates is constructed from fine (bottom) to coarse (top), such that each aggregate at a finer level may be associated to several larger aggregates at a subsequent coarser scale, with different weights. This soft weighted aggregation allows the algorithm to avoid pre-mature local decisions and to detect segments based on a global saliency measure. The algorithm is very fast, since only its initial stage operates at the level of individual voxels. It collects statistics of filter responses, identifies regions of coherent textures, quantifies the shape of segments, their boundaries and their density variability at all scales, etc., allowing the emergence of image segments according to any desired aggregation and saliency criteria. Moreover, each segment emerges from the aggregation process equipped with a list of accumulated numerical descriptors, its features, which can then be used for classification and diagnosis processes.

Multi-Channel and 3D Anisotropy: A major aspect of MRI is the wide variety of pulse sequences (modalities) available for producing different images. Each modality gives rise to a different image that may highlight different type of tissues. In this work segmentation was applied to a single T1 channel. However applying segmentation (and likewise classification) simultaneously to images obtained by several channels can lead to superior results that usually cannot be achieved by considering just one channel. Another important aspect is the anisotropic nature of most clinical MRI data (with lower vertical resolution) which if not taken into account may lead to inaccurate analysis of the data. Relying on the flexibility of the segmentation framework we applied a 3D multi-channel segmentation algorithm that can process several modalities simultaneously, and handle both isotropic data as well as anisotropic data.

\section{$2.2 \quad$ Feature Extraction}

The segmentation process computes statistical aggregative features throughout the pyramid construction. These features, which affect the formation of aggregates, are also available for the classification of anatomical structures at the end of the process. The development of the set of features is guided by interaction with expert radiologists, and the quantitative effects of the various features are determined by the automatic learning process described below. It can be shown that these properties can be calculated recursively (see [12, [14] for notations).

In this work, we expanded the set of features to include information about the expected location of the major tissue types. We incorporate the prior probability knowledge of anatomic structures using an MRI probabilistic atlas. We employ the International Consortium for brain mapping (ICBM) probability maps which 
represent the likelihood of finding GM, WM and CSF at a specified position for a subject that has been aligned to the atlas space. The ICBM452 atlas and brain data sets are brought into spatial correspondence using the Statistical Parametric Mapping (SPM) registration software [16] so that for every aggregate we can compute its average probability to belong to any of the three tissue categories.

Construction of the classifier based on these features requires consideration of the inter-subject and intra-subject variability; therefore all features were normalized for each brain. Table1 presents a partial list of the features for aggregate $k$ at scale $s$ used in this study.

Table 1. Aggregative features

Prior anatomical knowledge:

Average probabilities: denoted $\overline{P_{W M}}, \overline{P_{G M}}, \overline{P_{C S F}}$ which represent the average likelihood of finding WM, GM, and CSF in $k$ respectively. Intensity statistics:

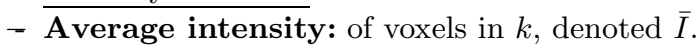

- Maximum intensity: maximal average intensity of sub-aggregates at scale 2 .

- Variance of average intensities: of sub-aggregates of $k$ at scale $r$.

- Intensity moments: averages of products of the intensity and the coordinates of voxels in $k$, denoted $\overline{I x}, \overline{I y}, \overline{I z}$.

Shape:

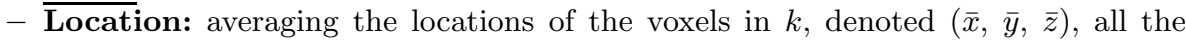
brains were spatially normalized to the same stereotaxic space using the SPM 16].

- Shape moments: the length, width and depth $(L, W, D$ respectively) calculated by applying principal component analysis to the covariance matrix of the aggregate. Neighborhood statistics:


border of aggregates $k$ and $l$.

- neighborhood average intensity: of aggregate $k$ defined as $\frac{\sum_{l} B_{k l} \bar{I}_{l}}{\sum_{l \neq k} B_{k l}}$

\subsection{Support Vector Machine (SVM) Classification}

We extract a candidate set of segments from the intermediate level of the pyramid (scales 5,6 from all 13 scales) which correspond to brain tissue regions. To construct the classifier we utilize "ground-truth" expert segmentation which is provided along with the real clinical brain MRI data. Generally we assume (1) having a training sample of $M$ candidate segments, Cand $=\left\{f_{1}, \ldots, f_{M}\right\}$, each is described by a d-dimensional feature vector (we normalize each of the features to have zero mean and unit variance), and (2) a mask indicating the voxels marked by an expert as GM, WM, CSF and background (BG). Since many of the candidate segments may contain a mixed collection of the categories, the labeling category is determined based on the category marked by the maximum number of voxels associated with the segment. 
Table 2. Our average classification measures. The table lists the mean $( \pm$ S.D $)$ classification measures obtained on all 20 subjects for the four different classes.

\begin{tabular}{|l|l|l|l|l|}
\hline Classes & Overlap & FP & $\kappa$ & $J$ \\
\hline \hline WM: & $0.80 \pm 0.04$ & $0.19 \pm 0.05$ & $0.80 \pm 0.04$ & $0.67 \pm 0.03$ \\
GM: & $0.86 \pm 0.04$ & $0.26 \pm 0.00$ & $0.81 \pm 0.04$ & $0.68 \pm 0.03$ \\
CSF: & $0.43 \pm 0.12$ & $0.25 \pm 0.20$ & $0.51 \pm 0.11$ & $0.35 \pm 0.12$ \\
BG: & $0.987 \pm 0.005$ & $0.003 \pm 0.002$ & $0.985 \pm 0.004$ & $0.992 \pm 0.002$ \\
\hline
\end{tabular}

Twenty normal MR brain data sets and their manual segmentations were provided by the Center for Morphometric Analysis at Massachusetts General Hospital and are available at "http://www.cma.mgh.harvard.edu/ibsr/". The sets are sorted based on their difficulty level. We separated the sets to "odd" and "even" indexes. The classifier was trained on the odd sets and tested on the even sets and vice versa, so that both the training and the testing consist of ten sets including the entire range of difficulty. The training data was used to construct an SVM-based classifier. The results presented here were obtained by using a radial basis function $\operatorname{RBF}$ kernel $\left(K(x, y)=e^{-\gamma|x-y|^{2}}\right)$. A detailed description of SVMs can be found in [17].

Following the learning phase, in the testing phase an unseen MRI scan is obtained. After segmentation and feature extraction we apply the SVM classifier to every candidate segment in the test set and finally assign a category-label to each candidate. All candidates segments are projected onto the data voxels using the segmentation interpolation matrix (see details in [12]). The maximum association weight of the voxel determines the segment which the voxel belongs to, which lead to an assignment of a class label to each voxel.

\section{Results}

The integrated approach was tested on 20 coronal T1-weighted real MRI data set of normal subjects with GM,WM and CSF expert segmentations provided by the Internet Brain Segmentation Repository (IBSR), after they have been positionally normalized. The brain scans used to generate these results were chosen because they have been used in published volumetric studies in the past and because they have various levels of difficulty. This allows the assessment of the methods performance under varying conditions of signal to noise ratio, INU, PVE, shape complexity, etc. We tested our approach using 45 central coronal slices which contain $0.94 \pm 0.02$ of the brain voxels including the cerebellum and brain stem.

The results presented were obtained by overlaying the candidate segments of the brain set tested according to their labeling category by the SVM classifier. The validation scores presented are based on the common measures for spatial overlap (e.g., 6], [19]). Denote by $(S)$ the set of voxels automatically detected as a specific class and $(R)$ the set of voxels labeled as the same class in the 'ground 
Table 3. Average J-scores for various segmentation methods on 20 brains

\begin{tabular}{|l|l|l|l|}
\hline Method: & WM & GM & CSF \\
\hline \hline Manual (4 brains averaged over 2 experts): & 0.876 & 0.832 & - \\
\hline Our Method: & $\mathbf{0 . 6 6 9}$ & $\mathbf{0 . 6 8 0}$ & $\mathbf{0 . 3 4 6}$ \\
\hline Pham and Prince [8]: & 0.7 & 0.6 & - \\
Shattuck et. al. [3]: & 0.595 & 0.664 & - \\
Zeng et. al. [7]: & - & 0.657 & - \\
Burkhardt et. al. [18] (trium): & 0.578 & 0.644 & 0.206 \\
adaptive MAP(amap) [2]: & 0.567 & 0.564 & 0.069 \\
biased MAP(bmap) 2]: & 0.562 & 0.558 & 0.071 \\
fuzzy c-means(fuzzy) [2]: & 0.567 & 0.473 & 0.048 \\
Maximum Aposteriori Probability (map) [2] $:$ & 0.554 & 0.550 & 0.071 \\
Maximum-Likelihood(mlc) 2] : & 0.551 & 0.535 & 0.062 \\
tree-structure k-means(tskmeans) 2]: & 0.571 & 0.477 & 0.049 \\
\hline
\end{tabular}

truth' reference. The classification measures used in Table 2 and 3 are defined as follows:

- Overlap: $|S \cap R| /|R|$.

- FP: $|S \cap \bar{R}| /|R|$.

- $\kappa$ statistics (Dice coefficient): $2|S \cap R| /(|S|+|R|)$

- Jaccard similarity $J:|S \cap R| /(|S \cup R|)$

Table 3 and Figure 1 display a quantitative comparison of our approach with ten other algorithms. Six of them are provided with the data [2]. We also included in Table 3 four additional studies which report the average results for part of the tasks $([3,7],[8,[18])$. The comparison is based on the $J$ metric score provided in these studies. Our average scores for all classes were comparative or superior to previously reported results, where we obtained a significance difference to other

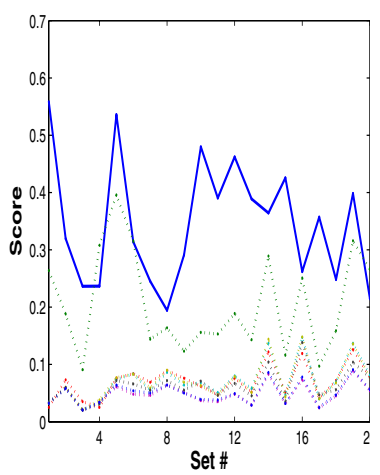

(a) Cerebrospinal Fluid (CSF)

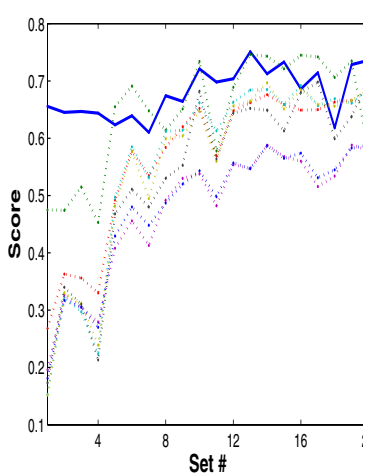

(b) Gray Matter (GM)

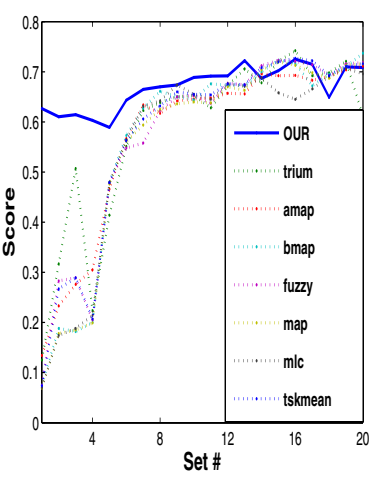

(c) White Matter (WM)

Fig. 1. Overlap scores between manual and automatic segmentations over 20 brain scans with decreasing levels of difficulty (from set index 1 to 20). Our results compared with seven other algorithms for the task of GM, WM, and CSF Detection. 


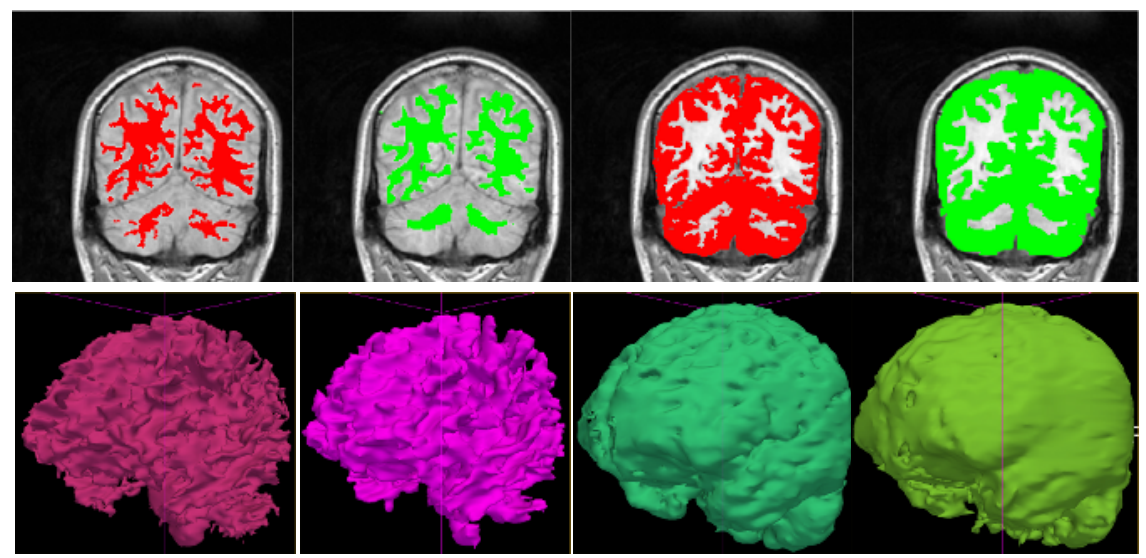

(a)WM-Ground-Truth(b)WM-Automatic(c)GM-Ground-Truth(d) GM-Automatic

Fig. 2. WM and GM identification. The upper row presents classification results projected on a $2 \mathrm{D} \mathrm{T} 1$ slice. The lower row demonstrates a $3 \mathrm{D}$ view of the results.

algorithms (for the GM and WM $p \leq 0.005$ ). The results are especially high on the most difficult cases (i.e. sets 1-5 see Fig. 1). Moreover, the other metrics presented in Table 2 show high detection rates for all categories identified. Figure 2 demonstrates the WM and GM segmentations produced by the method in a $2 \mathrm{D}$ and $3 \mathrm{D}$ view respectively.

\section{Discussion}

MRI is considered the ideal method for brain imaging. The 3D data and the large number of possible protocols allow to identify anatomical structures as well as abnormal brain structures. In this work we focus on segmenting normal brains into three tissues WM, GM and CSF. The segmentation pyramid provides a rich, adaptive representation of the image, enabling detection of various anatomical structures at different scales. A key aspect of the approach is the comprehensive set of multiscale measurements applied throughout the segmentation process. These quantitative measures, which take into account the atlas information, can further be used for clinical investigation. For classification we apply automatic learning procedure based on an SVM algorithm using data pre-labeled by experts. Our approach is unique since it combines a rich and tunable set of features, emerging from statistical measurements at all scales. Our competitive results, obtained using a standard SVM classifier, demonstrate the high potential of such features.

The algorithm was evaluated on real 3D MRI brain scans, demonstrating its ability to detect anatomical brain structures. It requires no restrictions from the MRI scan protocols and can further be generalized to other tasks and modalities, such as to detect evolving tumors or other anatomical substructures. Future work will expand the approach to detection of internal brain anatomical structures. Extraction of other classes of structures may require the use of additional features and perhaps a more detailed knowledge domain available in brain atlases. 
Acknowledgement. Research was supported in part by the Binational Science foundation, Grant No. 2002/254, by the Israel Institute of Technology and by the European Commission Project IST-2002-506766

Aim Shape. Research was conducted at the Moross Laboratory for Vision and Motor Control at the Weizmann Institute of Science.

\section{References}

1. Pham, D., Xu, C., Prince, J.: Current methods in medical image segmentation. Annual Review of Biomedical Engineering 2 (2000) 315-337

2. Rajapakse, J., Kruggel, F.: Segmentation of MR images with intensity inhomogeneities. IVC 16(3) (1998) 165-180

3. Shattuck, D.W., Sandor-Leahy, S.R., Schaper, K.A., Rottenberg, D.A., Leahy, R.M.: Magnetic resonance image tissue classification using a partial volume model. Neuroimage 13(5) (2001) 856-76

4. Bezdek, J.C., Hall, L.O., Clarke, L.P.: Review of MRI segmentation techniques using pattern recognition. Medical Physics 20(4) (1993) 1033-1048

5. Sonka, M.M., Fitzpatrick, J.M., eds.: Handbook of Medical Imaging. SPIE (2000)

6. Zijdenbos, A., Dawant, B.: Brain segmentation and white matter lesion detection in MRI. Critical Reviews in Biomedical Engineering 22 (1994)

7. Zeng, X., Staib, L.H., Schultz, R.T., Duncan, J.S.: Segmentation and measurement of the cortex from 3D MRI using coupled surfaces propagation. IEEE MI (1999)

8. Pham, D., Prince, J.: Robust unsupervised tissue classification in MRI. IEEE Biomedical Imaging: Macro to Nano (2004)

9. Wells, W.M., Grimson, W., Kikinis, R., Jolesz, F.A.: Adaptive segmentation of MRI data. IEEE MI 15 (1996) 429-442

10. Zhang, Y., Brady, M., Smith, S.: Segmentation of brain MRI through a hidden markov random field model and the expectation-maximization algorithm. IEEE Medical Imaging 20(1) (2001) 45-57

11. Van-Leemput, K., Maes, F., Vandermeulen, D., Colcher, A., Suetens, P.: Automated segmentation of MS by model outlier detection. IEEE MI 20 (2001) $677-688$

12. Akselrod-Ballin, A., Galun, M., Gomori, J.M., Fillipi, M., Valsasina, P., Brandt, A., R.Basri: An integrated segmentation and classification approach applied to multiple sclerosis analysis. CVPR (2006)

13. Sharon, E., Brandt, A., Basri, R.: Fast multiscale image segmentation. CVPR (2000) 70-77

14. Galun, M., Sharon, E., Basri, R., Brandt, A.: Texture segmentation by multiscale aggregation of filter responses and shape elements. ICCV (2003) 716-723

15. Brandt, A., McCormick, S., Ruge, J., eds.: Algebraic multigrid (AMG) for automatic multigrid solution with application to geodetic computations. Inst. for Computational Studies, POB 1852, Fort Collins, Colorado (1982)

16. Frackowiak, S., Friston, K., Frith, C., Dolan, R., Price, C., Zeki, S., Ashburner, J., Penny, W., eds.: Human Brain Function. Academic Press (2003)

17. Vapnik, V., ed.: The Nature of Statistical Learning Theory. Springer-Verlag (1995)

18. Burkhardt, J.: A markov chain monte carlo algorithm for the segmentation of t1-mr-images of the head. Diploma thesis, Technische Universitat Munchen (2003)

19. Gerig, G., Jomier, M., Chakos, M.: Valmet: A new validation tool for assessing and improving 3D object segmentation. MICCAI (2001) 516-523 